\title{
Surface integral equation analysis of the electromagnetic interference (EMI) impact of wind turbines on radar systems
}

\author{
D. Poljak, V. Dorić \& D. Čavka \\ University of Split, FESB, Croatia
}

\begin{abstract}
Wind turbines (WT), as large conducting structures, may disturb the use of radar in civil and military applications. Therefore, an efficient simulation of a radar system in the presence of a WT is required. This paper deals with WT impact to the radar system operation. The formulation is based on the corresponding surface integral equation (SIE), i.e. the electric field integral equation (EFIE) for metallic objects. The solution is carried out using the Boundary Integral Equation Method (BIEM) combined with physical optics (PO). The model aims to account for the rotation of the blades and to ensure corresponding radar cross section (RCS) prediction. Some illustrative computational examples arising from realistic scenarios are given in this paper. Keywords: wind turbines, radar systems, electromagnetic interference, electric field integral equation, physical optics, numerical solution.
\end{abstract}

\section{Introduction}

Due to a continuous need for clean and renewable energy the number of wind turbines (WTs) has been increasing in countries around the world. On the other hand, WTs are at typical radar frequencies large structures having a large radar cross-section (RCS). Consequently, due to the blade rotation, WTs may disrupt the use of radar in either civil or military applications [1].

Therefore, an efficient electromagnetic model to analyze the interaction between radar systems and WTs is necessary. The character of potential electromagnetic interference (EMI) depend on many parameters such as location and type of WT, physical and electrical properties of the blade, signal frequency, 
etc. To analyze the interaction between a WT and a radar system, the RCS of a WT has to be determined, i.e. the scattered electric field has to be evaluated. The field scattered from the metallic object is often determined by integrating the corresponding surface current density. The current density is, on the other hand, obtained by solving the surface integral equation (SIE), i.e the electric field integral equation (EFIE) numerically, mostly via boundary element method (BEM) or method of moments (MoM) [2].

However, EFIE can be numerically solved relatively efficiently via certain numerical technique, e.g. via MoM if a given conducting object is electrically small, or comparable to the wavelength. As WTs are electrically large structures some of the asymptotic numerical methods should be implemented. Thus, the present work features the use of physical optics (PO) [3] for the RCS assessment. In particular, this paper deals with bRCS-a of 3D model of a WT.

The paper is organized as follows: First, EFIE is derived. Then, some fundamental concepts of PO approximation and RCS assessment Furthermore, some illustrative computational examples are presented. The paper ends up with some concluding remarks.

\section{Integral equation formulation}

SIE, i.e EFIE can be obtained from Maxwell equations featuring the continuity conditions for the tangential field components.

From the first Maxwell equation it follows that a time harmonic electric field $\vec{E}$ can be expressed in terms of magnetic vector potential $\vec{A}$ and electric scalar potential $\varphi$ :

$$
\vec{E}=-\nabla \varphi-j \omega \vec{A}
$$

where $\omega$ stands for operating frequency.

Under certain conditions magnetic vector potential $\vec{A}(\vec{r})$ is given by the particular integral:

$$
\vec{A}(\vec{r})=\frac{\mu}{4 \pi} \iint_{V} \vec{J}\left(\vec{r}^{\prime}\right) \frac{e^{-j k\left|\vec{r}-\vec{r}^{\prime}\right|}}{\left|\vec{r}-\vec{r}^{\prime}\right|} d V\left(\vec{r}^{\prime}\right)
$$

where $\vec{J}$ is volume current density and $\mu$ is the medium permeability.

Furthermore, electric scalar potential $\varphi$ is given by the following integral:

$$
\varphi(\vec{r})=\frac{1}{4 \pi \varepsilon} \iint_{V} \rho\left(\vec{r}^{\prime}\right) \frac{e^{-j k\left|\vec{r}-\vec{r}^{\prime}\right|}}{\left|\vec{r}-\vec{r}^{\prime}\right|} d V\left(\vec{r}^{\prime}\right)
$$

where $\rho$ denotes the volume charge density and $\varepsilon$ is the medium permittivity. Note that $\left|\vec{r}-\vec{r}^{\prime}\right|$ is a distance from the source to the observation point, respectively. 
Applying the time-harmonic version of the continuity equation [4]:

$$
\nabla \vec{J}=-j \omega \rho
$$

the scalar potential becomes:

$$
\varphi(\vec{r})=-\frac{1}{j 4 \pi \omega \varepsilon} \iint_{V} \nabla^{\prime} \vec{J}\left(\vec{r}^{\prime}\right) \frac{e^{-j k\left|\vec{r}-\vec{r}^{\prime}\right|}}{\left|\vec{r}-\vec{r}^{\prime}\right|} d V\left(\vec{r}^{\prime}\right)
$$

For the case of perfectly conducting (PEC) bodies, volume current density is replaced by the surface current density $\vec{J}_{s}[2,4]$. Consequently, volume integrals (2) and (5) become:

$$
\begin{gathered}
\vec{A}(\vec{r})=\frac{\mu}{4 \pi} \iint_{S} \vec{J}_{S}\left(\vec{r}^{\prime}\right) \frac{e^{-j k\left|\vec{r}-\vec{r}^{\prime}\right|}}{\left|\vec{r}-\vec{r}^{\prime}\right|} d S\left(\vec{r}^{\prime}\right) \\
\varphi(\vec{r})=-\frac{1}{j 4 \pi \omega \varepsilon} \iint_{S} \nabla_{S}{ }^{\prime} \vec{J}_{S}\left(\vec{r}^{\prime}\right) \frac{e^{-j k\left|\vec{r} \vec{r}^{\prime}\right|}}{\left|\vec{r}-\vec{r}^{\prime}\right|} d S\left(\vec{r}^{\prime}\right)
\end{gathered}
$$

where $\nabla_{S}$ denotes the two-dimensional nabla operator.

Finally, combining (1), (6) and (7) yields the following representation of the scattered field:

$$
\vec{E}^{s c t}=-\frac{j \omega \mu}{4 \pi} \iint_{S} \vec{J}\left(\vec{r}^{\prime}\right) \frac{e^{-j k\left|\vec{r} \vec{r}^{\prime}\right|}}{\left|\vec{r}-\vec{r}^{\prime}\right|} d S\left(\vec{r}^{\prime}\right)+\frac{1}{j 4 \pi \omega \varepsilon} \iint_{S} \nabla_{S}{ }^{\prime} \vec{J}\left(\vec{r}^{\prime}\right) \frac{e^{-j k\left|\vec{r}-\vec{r}^{\prime}\right|}}{\left|\vec{r}-\vec{r}^{\prime}\right|} d S\left(\vec{r}^{\prime}\right)
$$

Furthermore, one obtains the SIE by satisfying the continuity conditions for the tangential field components:

$$
\vec{n} x \vec{E}_{\mid \tan }^{\text {tot }}=0
$$

where $\vec{n}$ is a unit vector normal to the surface PEC.

The total tangential field at the PEC surface can be written as a sum of incident and scattered field, respectively:

$$
\vec{E}_{\mid \tan }^{t o t}=\vec{E}_{\mid \tan }^{\text {inc }}+\vec{E}_{\mid \tan }^{\text {sct }}
$$

where

$$
\vec{E}_{\mid \tan }^{s c t}=-(j \omega \vec{A}+\nabla \varphi)_{\tan }
$$


Then SIE is obtained by combining (8)-(11) and is given by:

$$
\vec{E}_{\tan }^{i n c}=\frac{j \omega \mu}{4 \pi} \iint_{S} \vec{J}_{S}\left(\vec{r}^{\prime}\right) \frac{e^{-j k\left|\vec{r}^{\prime} \vec{r}^{\prime}\right|}}{\left|\vec{r}-\vec{r}^{\prime}\right|} d S\left(\vec{r}^{\prime}\right)-\frac{1}{j 4 \pi \omega \varepsilon} \iint_{S} \nabla_{S}{ }^{\prime} \vec{J}_{S}\left(\vec{r}^{\prime}\right) \frac{e^{-j k\left|\vec{r}-\vec{r}^{\prime}\right|}}{\left|\vec{r}-\vec{r}^{\prime}\right|} d S\left(\vec{r}^{\prime}\right)
$$

Once determining the surface current density it is possible to calculate the scattered field.

Utilizing the far field approximation condition [3]:

$$
r \geq \frac{2 D^{2}}{\lambda}
$$

where $r$ is a distance from the radiation source to the observation point, respectively, $D$ denotes the dimension of the radiation structure, and $\lambda$ is the signal wavelength.

The far-field approximation gives [3]:

$$
\vec{E}=-j \omega \vec{A}
$$

and the resulting relation for the scattered field is:

$$
\vec{E}^{s c t}=-\frac{j \omega \mu}{4 \pi} \iint_{S} \vec{J}_{S}\left(\vec{r}^{\prime}\right) \frac{e^{-j k\left|\vec{r}-\vec{r}^{\prime}\right|}}{\left|\vec{r}-\vec{r}^{\prime}\right|} d S\left(\vec{r}^{\prime}\right)
$$

Note that expression (15) is convenient for implementation within the physical optics (PO) approximation.

\section{Physical optics approximation}

Physical optics (PO) is an approximate method for determining surface currents assuming the surfaces to be locally planar and the reflection phenomena occur in accordance to the geometrical optics $[2,3]$.

It is worth mentioning that PO avoids the large matrix system and efficiently treats discontinuities and singularities in the high frequency problems.

\subsection{The calculation of the current density using physical optics}

In accordance to the geometrical optics, on a part of the object directly illuminated by an incident field, the induced current density is proportional to the incident magnetic field. On the part of the object in the shadow, the current density is assumed to be zero, i.e. it can be written $[2,3]$ : 


$$
\vec{J}_{s}=\left\{\begin{array}{c}
2 \vec{n} x \vec{H}^{i n c}, \text { for ilum. part } \\
0, \text { for shadow part }
\end{array}\right.
$$

where $\vec{H}^{i n c}$ is the magnetic field on the PEC object surface.

From the Maxwell equations it follows [2, 3]:

$$
\vec{H}^{i n c}=\frac{\vec{k} \times \vec{E}^{i n c}}{Z_{0}}
$$

where $Z_{0}$ is the free space impedance.

Within the far-field approximation (15) can be simplified, i.e. it follows:

$$
\vec{E}^{s c t}=-\frac{j \omega \mu}{4 \pi r} e^{-j k r} \iint_{S} \vec{J}_{S}\left(\vec{r}^{\prime}\right) e^{-j k \vec{r}^{\prime}} d S\left(\vec{r}^{\prime}\right)
$$

Relation (18) is applicable for objects having locally planar geometries.

Thus, PEC objects is discretised on triangular segments (Fig 1).

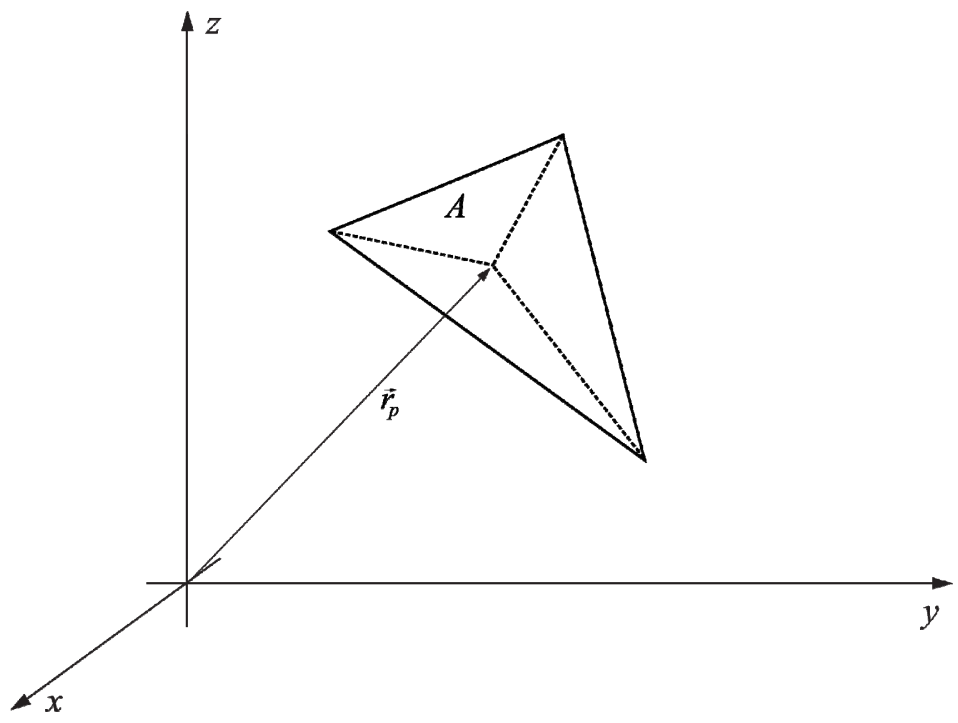

Figure 1: Triangular element geometry.

According to PO, on a triangular element surface (18) can be written, as follows: 


$$
\vec{E}_{A}^{s c t}=-\frac{j \omega \mu}{4 \pi r} e^{-j k r}\left|\vec{J}_{s}\left(\vec{r}^{\prime}\right)\right| \iint_{A} e^{j k\left(\vec{r}_{p} \cdot \vec{e}_{r}+\vec{r}_{p} \cdot \vec{r}_{i}\right)} d S\left(\vec{r}^{\prime}\right)
$$

where $A$ is the area of a triangle, $\vec{r}_{p}$ is a triangle coordinate, and $\vec{r}_{i}$ corresponds to the Poynting vector, i.e. the direction of the wave propagation.

Note that the current density is variable over the entire conducting structure and uniform over the segment.

\section{Assessment of radar cross section (RCS)}

Property of a conducting object to reflect a part of an incident field (as shown in Fig. 2), is defined by means of a radar cross section (RCS).
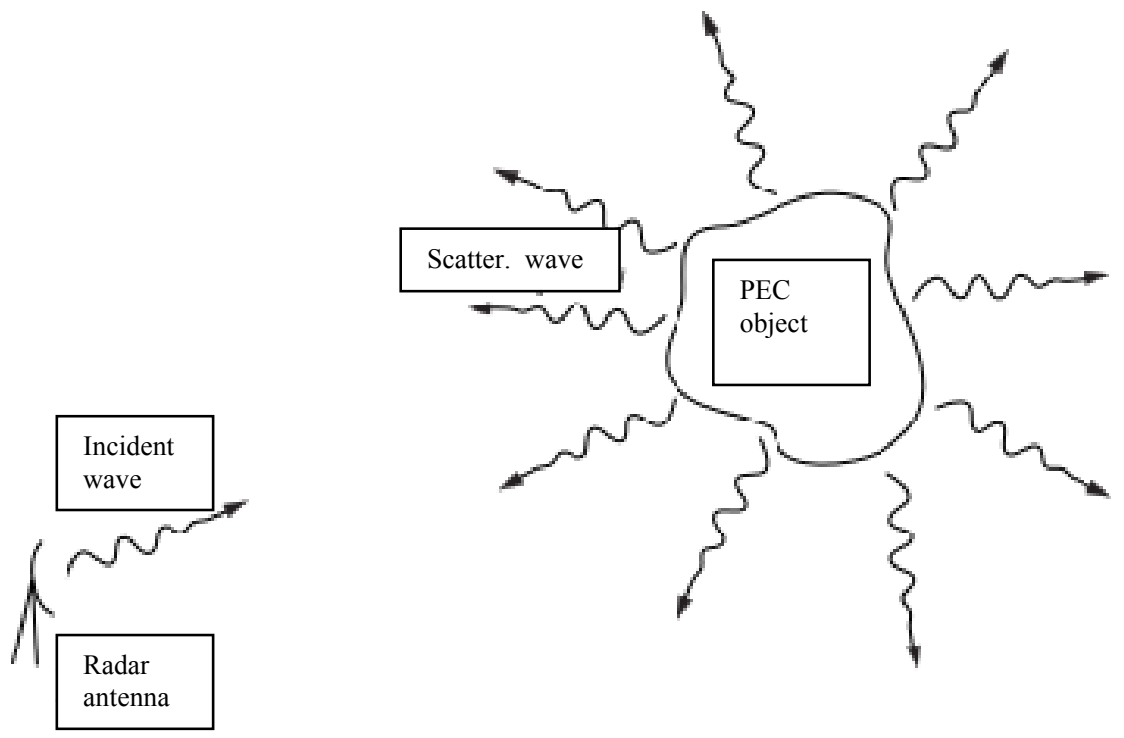

Figure 2: $\quad$ Scattering on a PEC object.

RCS of PEC objects gives the information how the object in reflects the radar ray in a given direction. The greater the RCS the greater is the reflected power of the radar signal. Generally, RCS depends on shape and size of the object same as polarisation, frequency and incidence angle of the radar signal [1].

Assuming the PEC object to be in the far field zone, radar signal can be approximated by the plane wave and RCS is defined by expression:

$$
\sigma=\lim _{r \rightarrow \infty} 4 \pi r^{2} \frac{\left|E^{s c t}\right|^{2}}{\left|E^{i n c}\right|^{2}}
$$


where $E_{s c t}$ is the scattered field, $E^{i n c}$ is the field incident to the PEC target, while $r$ represents the distance between the target and the radar. Relation (20) is also referred to as a monostatic RCS, as transmitting and receiving antenna of the radar system, respectively are assumed to be at the same location.

On the other hand, the bistatic radar cross section (bRCS), where transmitter and receiver are on different locations is defined by expression:

$$
\sigma(\phi, \theta)=4 \pi \frac{U(\phi, \theta)}{P_{d}(\phi, \theta)}=\lim _{r \rightarrow \infty} 4 \pi r^{2} \frac{\left|E^{s c t}(\phi, \theta)\right|^{2}}{\left|E^{\text {inc }}(\phi, \theta)\right|^{2}}
$$

For the case of monostatic radar the ratio of the received and emitted power, respectively, is given by the following radar equation:

$$
\frac{P_{r}}{P_{t}}=\frac{G^{2}}{4 \pi} \frac{\lambda^{2}}{\left(4 \pi r^{2}\right)^{2}} \sigma
$$

where $G$ stands for the antenna gain, $r$ is the distance from the radar antenna to the conducting object, $\sigma$ is RCS and $\lambda$ is the signal wavelength. form:

If the bistatic radar is of interest the corresponding radar equation is of the

$$
\frac{P_{r}}{P_{t}}=\frac{G_{1} G_{2}}{4 \pi} \frac{\lambda^{2}}{\left(4 \pi r_{1} r_{2}\right)^{2}} \sigma
$$

where $G_{1}$ and $G_{2}$ is the gain of transmitting and receiving antenna, respectively, while $r_{1}$ and $r_{2}$ are the corresponding distances from the antennas to the conducting object.

\section{Numerical results}

A three-dimensional computational model of WT is assembled in this work. Height of the tower is $78.8 \mathrm{~m}$ (from ground to the hub center), blade length (from the hub center to the tip) is $37.3 \mathrm{~m}$, diameter at the WT base and the top is $4.3 \mathrm{~m}$ and $2.345 \mathrm{~m}$, respectively. WT surface is discretised on 210,000 triangular elements. A part of the mesh is shown in Fig. 3.

Multiple (false) targets are assessed according to the scenario presented in Fig. 4. by:

Generally, a reflected signal delay $\Delta t$ (with respect to the direct ray) is given

$$
\Delta t=\frac{R_{1}+R_{2}-R}{c}
$$

Due to the Interrogator Side Lobe Suppression (ISLS) transponder is checked for $\Delta t=35 \mu$ s after receiving the first (direct) signal.

A distribution of points in which signal reflected from WT is detected by the receiver over the sphere of radius $5250 \mathrm{~m}$, where the signal level exceeds 


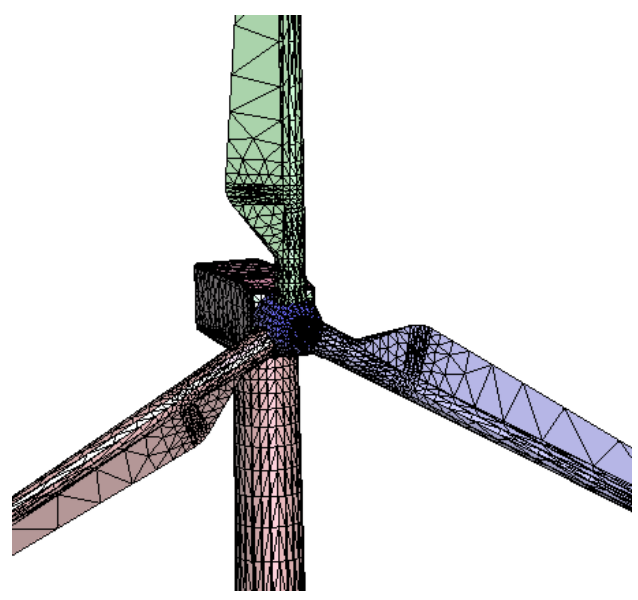

Figure 3: Discretisation with triangular elements (CAD model of a WT).

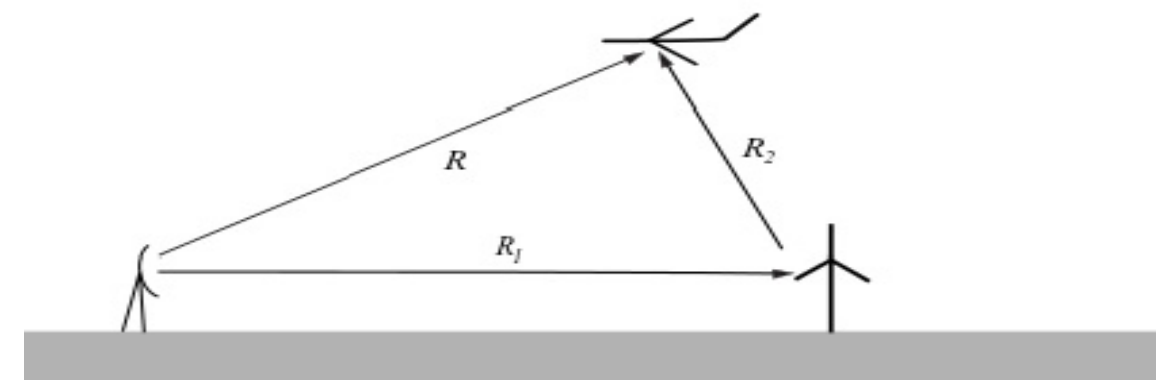

Figure 4: Direct and reflected signal.

the transponder sensitivity threshold of $(-77 \mathrm{dBm})$ and where false echo is possible is shown in polar coordinates in Fig 5(a). Figure 5(b) shows the same zones in rectangular coordinates.

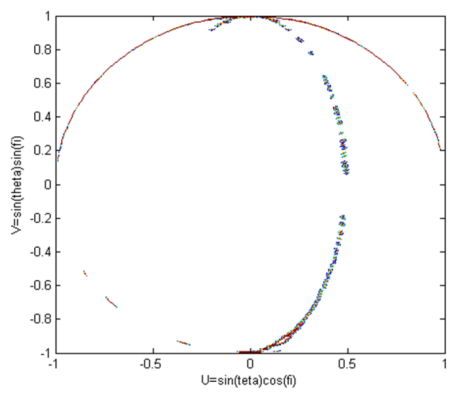

(a)

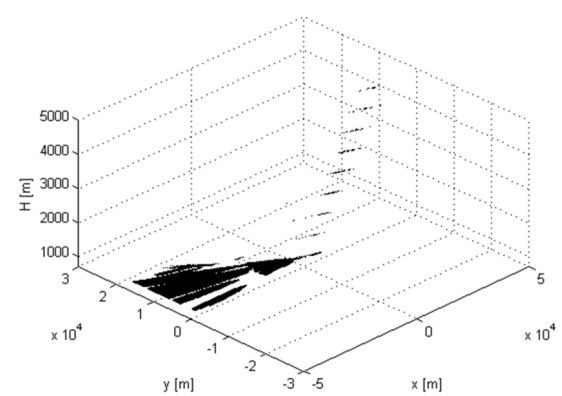

(b)

Figure 5: Zone of points in which signal reflected from WT is detected by the receiver: (a) Polar coordinates; (b) Rectangular coordinates. 
Interferences may also occur in downlink communications (as shown in Fig. 6).

Figure 7 shows the points in which radar could detect signal from the plane as a noise.

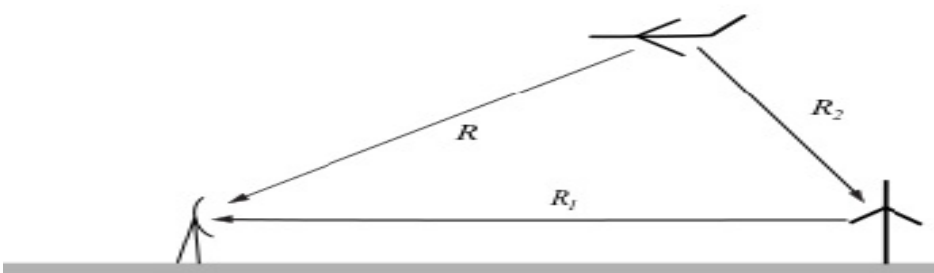

Figure 6: Reflected signal in a downlink with SSR (secondary surveillance radar).

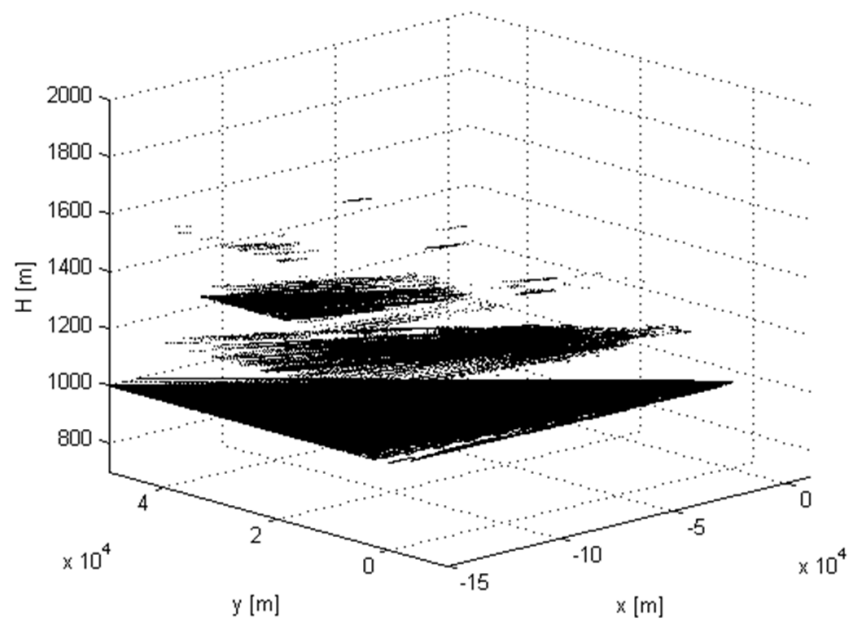

Figure 7: A three-dimensional representation of the airplane location where the radar system detects the reflected signal.

If SSR detects reflected signal with relatively small delay $(\Delta t<35 \mu s)$ still does not mean that an error in the assessment of the airplane direction will.

\section{Conclusion}

Analysis of wind turbine impact to the radar system operation is carried out in this work. The formulation is based on the corresponding surface integral equation (SIE) for conducting objects. The numerical solution is carried out 
using the Boundary Integral Equation Method (BIEM) combined with Physical Optics (PO) approximation. Knowing the induced current density over the entire object surface and related scattered field provides the calculation of Radar Cross Section (RCS). Some illustrative numerical results are presented in the paper.

\section{References}

[1] J.C.G. Matthews, C. Sarno, R. Herring, Interaction Between Radar Systems and Wind Farms, 2008 Loughborow Antennas \& Propagation Conference, Loughborrow, UK, March 2008.

[2] M. Jin, Theory and Computation of Electromagnetic Fields, IEEE Press Wiley, New Jersey, USA, 2010.

[3] Yamashita (Ed), Analysis Methods for Electromagnetic Wave Propagation, Artech House, London, UK, 1996.

[4] D. Poljak, Advanced Modeling in Computational Electromagnetic Compatibility, Wiley-Interscience, New Jersey, USA, 2007. 